\title{
DIAGNOSTIC AND THERAPEUTIC THORACIC SURGERY IN LEUKEMIA AND SEVERE APLASTIC ANEMIA
}

James M. Habicht, MD $^{\mathrm{a}}$

Alois Gratwohl ${ }^{\mathrm{b}}$

Michael Tamm, $\mathrm{MD}^{\mathrm{b}}$

Jürgen Drewe, $\mathrm{MD}, \mathrm{PhD}^{\mathrm{b}}$

Michael Proske, $\mathrm{MD}^{\mathrm{c}}$

Peter Stulz
Background: Pulmonary complications often occur in patients with leukemia and severe aplastic anemia. Particularly in patients having undergone bone marrow transplantation, respiratory diseases account for a significant number of deaths. In a retrospective study, we evaluated 41 patients with leukemia and severe aplastic anemia who were operated on consecutively from 1980 to 1995. Methods: Fourteen open lung biopsies, four videoassisted lung biopsies, and 24 lung resections were performed in 24 male and 17 female patients. Mean age was 32.2 years. Results: Eleven (27\%) early deaths occurred (30-day mortality): ten in patients after lung biopsy $(56 \%)$ and one after lung resection $(4 \%)(p<0.001)$. Perioperative morbidity relating to pulmonary disease or operation included $10(24.4 \%)$ cases of prolonged ( $>24$ hours) postoperative mechanical ventilation and two $(4.8 \%)$ cases of bleeding or hematoma. In one $(2.4 \%)$ patient a slowly developing, contained bronchial stump insufficiency appeared after lobectomy, which was successfully operated on 3 weeks later. Conclusion: We conclude that resection of localized pulmonary lesions, be it for diagnostic or therapeutic (or combined) purposes, can be carried out with low morbidity and mortality in patients with leukemia and severe aplastic anemia. However, early mortality is high after open or thoracoscopic lung biopsies in patients with acute-onset diffuse pulmonary disease, and little therapeutic benefit is realized in these cases. (J Thorac Cardiovasc Surg 1997;113:982-8)
$T^{\mathrm{h}}$ he number of patients with acquired forms of immunosuppression has risen continuously over the past two decades as a result of modern medical therapy of previously intractable diseases, ${ }^{1}$ solid organ transplantation, and bone marrow transplantation. In the course of their illness many of these patients have pulmonary complications usually caused by one of five pathogenetic mechanisms ${ }^{1}$ : (1) extension of the underlying disease process to involve the lungs, (2) opportunistic infection(s), (3) pulmonary reaction to therapy (drugs, irradiation, bone marrow transplantation), (4) a new, unrelated

From the Cardiothoracic Surgical Unit, Department of Surgery, Department of Medicine, ${ }^{b}$ and Department of Radiology, University Hospital, Basel, Switzerland.

Received for publication May 15, 1996; revisions requested July 2, 1996; revisions received Dec. 31, 1996; accepted for publication Jan. 2, 1997.

Address for reprints: James M. Habicht, MD, Cardiothoracic Surgical Clinic, University Hospital Basel, CH-4031 Basel, Switzerland.

Copyright (C) 1997 by Mosby-Year Book, Inc.

$0022-5223 / 97 \$ 5.00+0 \quad \mathbf{1 2 / 1 / 8 0 2 3 6}$ disease process (i.e., cardiac pulmonary edema or emboli), and (5) any combination of the aforementioned items. It is often impossible to obtain a reliable diagnosis on clinical and radiologic grounds alone, and sputum culture is known to be of limited value. Invasive diagnostic procedures such as bronchoscopy and bronchoalveolar lavage (BAL) are advocated to begin effective treatment. However, a negative result of BAL does not exclude infection, and the need for histologic examination of lung tissue or resection of diseased tissue may arise. The role of diagnostic and therapeutic surgery in patients with leukemia or aplastic anemia also remains a subject of controversy.

The aim of this study was to analyze our series of patients to assess mortality and morbidity, diagnostic and therapeutic benefit, and to attempt to define future guidelines for surgery.

\section{Patients and methods}

The investigated population comprised 41 consecutive patients with hematologic diseases and pulmonary complications who underwent diagnostic and therapeutic tho- 
racic operations from January 1980 to December 1995. Mean age was 32.2 years (standard deviation [SD] 16.8 years, ranging from 6 to 69 years). Twenty-four patients were male and 17 were female. Thirty-two had leukemia, and nine had severe idiopathic aplastic anemia. Before the operation, treatment of the hematologic disease had been bone marrow transplantation in 18 , chemotherapy in 15 , and antilymphocyte globulin in eight patients (Fig. 1).

A detailed retrospective analysis of hospital records and review of all chest x-ray films, thoracic computed tomographic scans, and histologic sections was performed. The patients were treated in the hemato-oncologic isolation department under prospectively recorded standardized conditions according to defined protocols for hematologic malignant diseases or severe idiopathic aplastic anemia. $^{2,3}$

Twenty-three parameters including the following were investigated: sex, age, diagnosis and therapy of hematologic disease, results of preoperative sputum and BAL studies, preoperative chest roentgenography and computed tomographic scanning, histologic and microbiologic assessment of lung tissue specimens, type of operation, granulocyte and platelet counts, change of medication after operation, intraoperative blood loss, administration of blood, perioperative (30-day) mortality, postoperative morbidity, and certain intervals such as occurrence of first respiratory symptoms until date of operation and occurrence of first radiologic changes until operation.

A radiologically diffuse pattern was defined as changes on chest $\mathrm{x}$-ray films and computed tomographic scans affecting all lobes of both lungs. A localized pattern did not necessarily indicate existence of just one single lesion. A gathering of a few spherical or patchy delineated lesions was also classified as localized, so long as the changes did not occur in all lobes.

Results of preoperative sputum and BAL studies were classified as corresponding or not corresponding to the results from tissue specimens. For instance, if sputum was negative for pathogenic bacteria and both histologic and microbiologic lung tissue examinations were also negative, the result would be described as corresponding. Sputum positive for pathogens but without confirmation in lung tissue was classified as not corresponding, as was negative sputum with detection of microbial agents in the tissue probe.

Specific histologic changes comprised results of sections and microbiologic investigations of lung specimens disclosing pneumonitis with a causative bacterial, viral, or fungal microorganism, as well as diagnoses such as pulmonary leukemic infiltration and pulmonary embolism. The designation nonspecific histology covered noninfectious inflammatory diseases such as idiopathic interstitial pneumonitis (Table I). We distinguished between specific and nonspecific histologic characteristics, because this distinction would directly influence effectiveness of managing the respiratory disease.

Changes in medication after results had been obtained from tissue specimens were divided into the categories no change or nondirected change versus directed change. For example, new antibiotic treatment in accordance with pathogen sensitivity and the ability to stop antibiotic treatment were defined as a directed change. In contrast,

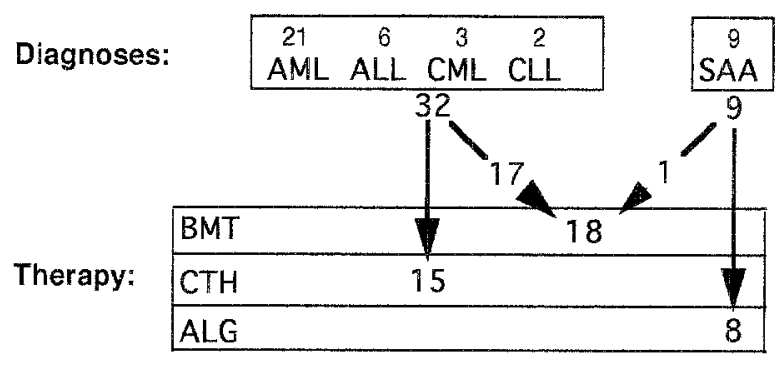

Fig. 1. The series comprised 41 patients, with a mean age of 3.2 years (range 6 to 69 years). Twenty-four patients were male (mean age 31.0 years) and 17 were female (mean age 33.4 years). $C T H$, Chemotherapy; $A L G$, antilymphocyte globulin; $B M T$, bone marrow transplant; $S A A$, severe aplastic anemia; $A M L$, acute myelogenous leukemia; $A L L$, acute lymphocytic leukemia; $C M L$, chronic myelogenous leukemia; $C L L$, chronic lymphocytic leukemia.

empirical change in antibiotic treatment that did not correspond to sensitivity testing was classified a nondirected change.

Statistical analysis was carried out by one of us (J.D.) with SPSS for Windows, version 7.0 (SPSS, Inc., Chicago, Ill.). Comparisons of proportions were performed by the two-tailed $\chi^{2}$ test or Fisher's exact test, as appropriate. Continuous parameters were first tested for normal distribution of data by the Shapiro-Wilk test. Depending on the results, analysis of variance was used for normally distributed data and the Mann-Whitney test otherwise. Effect size was described by odds ratio (OR) and corresponding $95 \%$ confidence intervals $(\mathrm{CI})$.

\section{Results}

Operations. A total of 42 diagnostic $(n=18)$ and therapeutic ( $n=24)$ lung resections were performed. Fourteen open lung biopsies were performed through small anterolateral thoracotomies and four video-assisted thoracoscopic (VATS) wedge biopsies were done. Four of these patients were already receiving mechanical ventilatory support before the operation. Therapeutic resections comprised 17 lobectomies, one bilobectomy, one segmentectomy, and five single or multiple wedge resections, one patient having undergone bilateral staged wedge resections.

Histology and preoperative diagnostic procedures. An overview of the histologic results and their relation to radiologic pattern and type of operation is presented in Table I. The most frequent findings were fungal infections $(14 / 21$ or $66.7 \%)$ in the group with specific histologic findings and idiopathic interstitial pneumonitis $(13 / 21$ or $61.9 \%)$ in those with nonspecific histologic findings. 


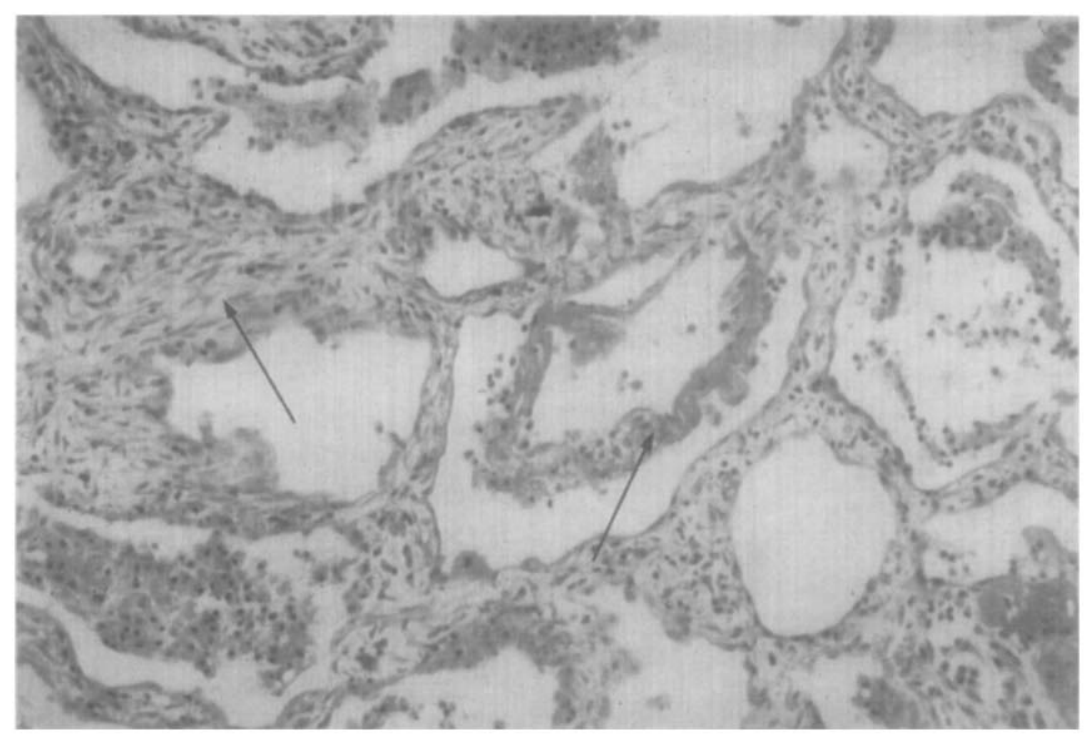

Fig. 2. Interstitial pneumonitis (hematoxylin and eosin stain; original magnification $\times 115$ ) with fibrosing alveolitis. Note widened alveolar septa containing fibrocytes (left arrow) and associated intraalveolar hyaline membranes with fibrin exudate and macrophages (center arrow).

Table I. Relation between histology, radiologic pattern, and type of operation ( $n=42$ interventions)

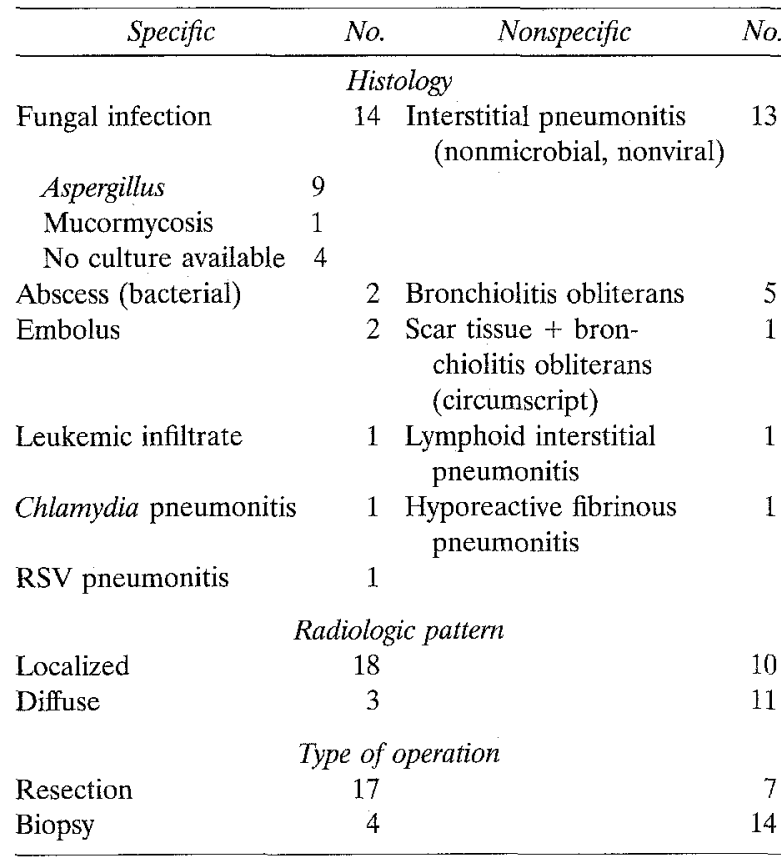

$R S V$, Respiratory syncytial virus.

Results from preoperative sputum specimens were accessible in 27 patients. In cases in which microorganisms were responsible for pulmonary disease, the microorganisms were correctly detected in only $8.3 \%(1 / 12)$ and no fungal infection was diag- nosed beforehand by examination of sputum. Secretion specimens were obtained with a bronchoscope and BAL was performed in a total of 28 patients. Causative microorganisms were correctly identified in only $36 \%(4 / 11)$. Results of the aforementioned investigations corresponded to results of tissue analysis in $44.5 \%(12 / 27)$ of sputum studies and in $57.1 \%$ $(16 / 28)$ of bronchoscopic examinations such that no causative microorganisms could be detected in either examination.

Preoperative fine needle biopsies and transbronchial biopsies were performed in three patients and one patient, respectively. The fine needle biopsies resulted in nonusable samples in two patients and a corresponding result in one patient with interstitial pneumonitis. Results of transbronchial biopsy showed suspected bronchiolitis obliterans in one patient before the operation, which was confirmed by VATS biopsy.

Mortality. Total early postoperative mortality (30day) amounted to $26.8 \%$ (Table II). All but two deaths were due to respiratory failure caused by the pulmonary disease; the other two patients died of acute leukemic relapse and intracerebral hemorrhage.

Statistical analysis showed the early mortality rate in patients with a diffuse radiologic pattern to be significantly higher than that of patients with a localized pattern. The same was true of patients having lung biopsies and of patients requiring me- 
The Journal of Thoracic and

Cardiovascular Surgery

Habicht et al. 985

Volume 113, Number 6

Table II. Postoperative mortality and morbidity

\begin{tabular}{|c|c|c|c|c|}
\hline & Total & Resection & Biopsy & $p$ Value \\
\hline Mortality (30-day) & $11 / 41(26.8 \%)$ & $1 / 23(4.3 \%)$ & $10 / 18(55.6 \%)$ & $<0.001$ \\
\hline \multicolumn{5}{|l|}{ Morbidity } \\
\hline Postop. ventilation $>24 \mathrm{hr}$ & $10 / 42(23.8 \%)$ & $1 / 24(4.2 \%)$ & $9 / 18(50 \%)$ & 0.012 \\
\hline Pneumothorax* & $2 / 42(4.8 \%)$ & $0 / 24$ & $2 / 18(11.1 \%)$ & 0.201 \\
\hline Bleeding $\dagger$ & $2 / 42(4.8 \%)$ & $1 / 24(4.2 \%)$ & $1 / 18(5.6 \%)$ & 0.999 \\
\hline Bronchial stump insufficiency & $1 / 42(2.4 \%)$ & $1 / 24(4.2 \%)$ & $0 / 18$ & 0.999 \\
\hline Intraop. blood loss (ml) $\%$ & $298(\mathrm{SD} 316)$ & $425(\mathrm{SD} 327)$ & 92 (SD 169) & $<0.001$ \\
\hline Intraop. blood (units) $\ddagger$ & $0.6(\mathrm{SD} 0.8)$ & $1.0(\mathrm{SD} 0.9)$ & $0.2(\mathrm{SD} 0.4)$ & 0.002 \\
\hline Blood until second postop. day (units) $\ddagger$ & $1.8(\mathrm{SD} 1.5)$ & $2.1(\mathrm{SD} 1.4)$ & $1.4(\mathrm{SD} 1.6)$ & 0.067 \\
\hline
\end{tabular}

* Pneumothorax requiring secondary drainage.

$†$ Bleeding requiring intervention or secondary drainage.

\$Data represent mean and standard deviation (SD).

chanical ventilation before the operation (Table III).

In patients with leukemia the early mortality was $31.2 \%(10 / 32)$ versus $11.2 \%(1 / 9)$ in patients with severe aplastic anemia $(p=0.401$; OR 3.64; CI 0.40 to 33.12). Early mortality in patients after bone marrow transplantation was significantly higher $(44.5 \%, 8 / 18)$ than in patients with aplastic anemia who were treated with antilymphocyte serum $(0 / 8)$ $(p=0.031$; OR $\infty$; CI 1.3 to $\infty)$. In patients undergoing biopsies, the mean duration of clinical symptoms such as fever, cough, and dyspnea was 4.3 weeks (SD 45 weeks) in survivors versus 2.4 weeks (SD 1.4 weeks) in nonsurvivors $(p=0.322)$. Mean interval from appearance of radiologic changes to operation was 23 days (SD 40.5 days) in survivors versus 7.4 days ( $\mathrm{SD} 8.7$ days) in nonsurvivors $(p=$ 0.528 ). These intervals, although not statistically significant, reflect a more rapid progression of respiratory distress in nonsurvivors. In patients with directed change in medication after the operation, the early mortality was $15.8 \%(3 / 19)$ as opposed to $36.4 \%(8 / 22)$ in patients without any (or nondirected) change in medication ( $p=0.173$; OR 0.33 ; CI 0.07 to 1.48 ).

All late deaths $(n=12)$ were due to a relapse of hematologic disease or complications of chemotherapy and, in patients who had had bone marrow transplantation, usually graft-versus-host reactions. At end of the study, 18 of the 41 patients $(43.9 \%)$ were long-term survivors; their mean survival time amounted to 47.5 months (ranging from 1 to 148 months)

Morbidity. One day before the operation the platelet count was $99.7 \times 10^{3} / \mu 1$ with $32 \%(13 / 41)$ of patients having counts under $40 \times 10^{3} / \mu \mathrm{I}$. However $56 \%(23 / 41)$ of patients had already received plate-
Table III. Parameters associated with early (30-day) mortality

\begin{tabular}{lrrrrr}
\hline & No. & Deaths & OR & CI & p Value \\
\hline Radiologic pattern & & & & & \\
$\quad$ Diffuse & 14 & 9 & 22.5 & $3.7-137.2$ & 0.0002 \\
$\quad$ Localized & 27 & 2 & & & \\
$\quad \begin{array}{l}\text { Type of operation } \\
\quad \text { Lung biopsy }\end{array}$ & 18 & 10 & 27.5 & $3.0-250.5$ & 0.0003 \\
$\quad$ Resection & 23 & 1 & & & \\
Mechanical ventilation" & & & & & \\
$\quad$ Yes & 4 & 4 & $\infty$ & $3.0-\infty$ & 0.003 \\
No & 37 & 7 & & & \\
\hline
\end{tabular}

$O R$, Odds ratio; $C I, 95 \%$ confience interval.

*Before operation.

lets at that time. Mean granulocyte count was $4.7 \times$ $10^{3} / \mu \mathrm{l} 1$ day before the operation. Seventy-three percent (30/41) of patients had counts under 3000/ $\mu l$, which reflects a high number of patients in whom aplasia was the result of chemotherapy or idiopathic aplastic anemia. Intraoperative blood loss (Table II) was significantly lower in the biopsy group $(92 \mathrm{ml})$ than in the resection group $(425 \mathrm{ml})$. However, mean preoperative platelet count was also significantly higher for the biopsy group $\left(137.9 \times 10^{3} / \mu \mathrm{l}\right.$; SD $\left.99 \times 10^{3} / \mu \mathrm{l}\right)$ than for the resection group $\left(70.9 \times 10^{3} \mu \mathrm{l}\right.$; SD $\left.85.2 \times 10^{3} / \mu 1, p=0.024\right)$, possibly influencing blood loss also. The amount of blood administered during the operation was significantly less in the biopsy group ( 0.2 units) than in the resection group (1.0 units); the amount administered after the operation up to the second postoperative day, however, was not significantly higher. Further morbidity related to the operation and necessitating operative intervention (hematoma, bleeding) was seen in two patients $(4.8 \%)$. None of the patients underwent rethoracotomy for postoper- 
ative bleeding. Ten patients $(24.4 \%)$ also needed prolonged ( $>24$ hours) postoperative mechanical ventilation. All except one of these patients had had biopsies. One patient $(2.4 \%)$ had a slowly developing, contained bronchial stump insufficiency after right upper lobectomy, which was successfully operated on 3 weeks later (see Table II).

\section{Discussion}

Severe aplastic anemia and leukemia are lethal illnesses necessitating the use of extraordinary therapeutic modalities, and pulmonary complications are numerous. Surgeons may be hesitant when dealing with these patients. First of all, the poor prognosis of the hematologic disease may deter them from undertaking a potentially therapeutic operation. In addition, the physical condition of the patients, involving risk factors such as coagulation disorders, thrombocytopenia, and predisposition to infectious complications, as well as prolonged postoperative intensive care, may give rise to legitimate concerns regarding the appropriateness of thoracic surgery. The aim of this study was to investigate whether the risk of surgery is justified in view of early mortality, morbidity, and therapeutic benefit in all or certain subgroups of patients with hematologic diseases.

The data presented show that it is important to distinguish between biopsies and resections. This may seem self-evident at first. However, in contrast to what one would expect, larger operations such as lobectomies have a significantly lower mortality $(4.3 \%)$ than open and VATS lung biopsies $(55.6 \%)$. This strongly suggests that neither the extent of lung tissue resection nor the risk of bleeding, infectious complications, or other aspects of operative trauma are definitive causes of early mortality, but rather the degree of respiratory impairment caused by the pulmonary disease itself.

Diffuse disease. The majority of lung biopsies were done in patients with radiologically diffuse changes, and histologic examination often revealed nonspecific inflammatory reactions (see Table I) such as idiopathic interstitial pneumonitis. The patients already receiving mechanical respiratory support before the operation also belonged to this category. Clinically manifest interstitial pneumonitis (see Fig. 2) is a widely known complication of radiotherapy, chemotherapy, and bone marrow transplantation, ${ }^{4,5}$ which occurs in up to $40 \%{ }^{5}$ of patients and may have a $50 \%$ mortality. ${ }^{1}$ Pathogenesis is multifactorial, and radiation as well as cyto- toxic drugs, viral infections, and other factors such as mismatched grafts and cyclosporine A play a role. Cytomegalovirus infection is a frequent finding in interstitial pneumonitis in some studies. ${ }^{1}$ However, we could not find a single case of documented cytomegalovirus pneumonitis in our histologic sections, possibly because the patients who had had bone marrow transplantation in this population were receiving antiviral medication and cytomegalovirus-negative blood products before the lung biopsy. Furthermore, this treatment was usually also combined with broad-spectrum empiric antimycotic, antibacterial, and steroid medication.

The presented results and those of others ${ }^{6,7}$ have shown a high early mortality of up to $62 \%$ after lung biopsies in patients with hematologic disorders, usually as a result of respiratory failure. In patients with diffuse disease it is not clear that even rapid identification of the cause after the beginning of respiratory compromise will lower the mortality. ${ }^{7,8}$ Some authors found patients with open lung biopsies to have an even worse outcome than patients without lung biopsy, and it has been speculated that this outcome might be related to morbidity of the procedure itself. ${ }^{6,7}$ However, in our experience surgical morbidity and respiratory impairment caused by small anterolateral thoracotomies or VATS procedures are limited. Moreover, we found a slightly higher frequency of directed change $(21 / 41 ; 51 \%)$ in therapy after biopsy than the $10 \%$ to $20 \%$ substantive changes stated by others. ${ }^{7}$ New antibiotic treatment after detection of a specific microbial organism constitutes a directed change in therapy; however, the possibility to stop a broad-spectrum empirical regimen of antibiotic or tuberculostatic medications and to begin high-dose steroids in proven noninfectious inflammatory reactions must be equally termed a substantial or a directed change in therapy. Interpretation is even more complex, because no change in therapy does not necessarily mean that a biopsy was futile. No change can also mean that a biopsy was successful by confirming a correct treatment begun before the operation. Whether "successful" biopsies then can alter the clinical course remains to be seen, but after reviewing our data we believe that open or VATS biopsies in diffuse pulmonary disease can be of value in certain instances. Indications must be evaluated in each individual case. Although the difference did not reach statistical significance, it is important to distinguish between patients with acute-onset, diffuse pulmonary disease with rapidly progressing 
respiratory distress and those with late-onset disease with a slow clinical course. In the latter, lung biopsies are often of diagnostic and therapeutic use, and the VATS technique with one-lung ventilation can be chosen. We found some cases of bronchiolitis obliterans and lymphoid interstitial pneumonitis, both of which are speculated to be graft-versus-host reactions in patients who have had bone marrow transplantation, ${ }^{9,10}$ as well as one case of respiratory syncytial virus pneumonitis. In acute-onset disease the interventions are most often in vain, especially when broad-spectrum empiric therapy has already been administered. In those patients already intubated and receiving mechanical ventilation the VATS approach does not offer much advantage with regard to pain management. Also, changing from a single-lumen to a double-lumen tube and one-lung ventilation with $100 \%$ oxygen may jeopardize the well-being of patients with severe respiratory insufficiency or aggravate preexisting pulmonary hypertension. Whenever the decision needs to be made to perform a biopsy in patients with acute onset and rapid progression of respiratory distress, this should be done expeditiously and before empiric medication is begun.

Localized disease. Focal lung lesions after bone marrow transplantation have been demonstrated to be fungal in up to $78 \%$ of patients. ${ }^{11}$ In the presented population of patients with leukemia and aplastic anemia having undergone resections of radiologically localized disease, $58 \%$ proved to have fungal infections, the predominant agent being $A s$ pergillus (see Table I). Only one patient had radiologic evidence of cavitary disease. The usual finding was a mass or localized infiltrate. Histologically, aspergillosis was invasive, which is a known finding in patients with neutropenia and differs from classic aspergilloma. ${ }^{12}$ Over the past few years several positive reports on therapeutic surgery for this indication have been published, ${ }^{12-15}$ and our results confirm this. Doubts remain, however, whether surgical treatment is necessary or whether antimycotic treatment alone is sufficient. One argument in favor of resection is that mycotic infections in patients with neutropenia usually show a tendency to grow under full antimycotic treatment, because these medicaments are not effective enough owing to insufficient cellular defense and limited diffusion of drugs in necrotic tissue. If aplasia is severe and long-lasting, as in the case of severe idiopathic aplastic anemia, conservative treatment may have unsatisfactory results with patients dying of dissem- ination or complications of invasion (i.e., hemorrhage). If aplasia is transitory, as in the case of patients with leukemia after a chemotherapeutic cycle, it can be argued that a fungal infection occurring at the end of an aplastic episode may heal with medical therapy alone inasmuch as the cellular reaction will continuously increase. However, the low mortality and morbidity of resections observed in this study suggest that the risk of waiting is higher than the risk of operating. In our patient population no recurrence of mycotic disease was observed in the lung or elsewhere after resections, which were usually therapeutic and complete in radiologically localized disease. Another important argument in favor of surgery is that preoperative diagnosis is often lacking or uncertain. In our population mycotic infection was confirmed by bronchoscopic investigations in only $25 \%(2 / 8)$ of patients before the operation. Sputum was negative for pathogens in all patients with fungal infection. Furthermore, we deem percutaneous needle aspirations in patients with aplastic anemia to be dangerous, because we have seen catastrophic hemorrhage with lethal outcome.

Platelets and if necessary fresh frozen plasma are administered before and during the operation, with the aim of raising platelet counts to $40,000 /$ $\mu 1$. Mean intraoperative blood loss is low for resections, and the amount of blood administered during the first 2 days after the operation is not significantly higher in patients having resections than in those having biopsies. This suggests that intraoperative hemorrhage or continuous postoperative oozing is rare. Hence, for diagnostic and therapeutic reasons, we advocate that resection of single or few ipsilateral delineated lesions developing or persisting despite antibiotic and antimycotic therapy be carried out as early as possible. Rarely, we have even done this in patients with hematologic disorders who had no clinical symptoms. In contrast, if there are a multitude of bilateral lesions and the patient's condition deteriorates rapidly, operation is not indicated and an attempt at control with high-dose antifungal agents should be undertaken. In such cases mycotic infections will often spread systemically and no effective therapy remains. However, one patient in this series with a good clinical course and general condition was able to undergo staged excisions bilaterally, with the first resection yielding necrotic lung tissue invaded by Aspergillus and the subsequent contralateral resections yielding 
nonspecific scar tissue, probably resulting from resolved mycotic infiltrates.

\section{Conclusions}

In patients with hematologic disorders who have pulmonary complications, early mortality and morbidity of lung resections and biopsies are usually due to the pulmonary disease itself, not to the extent or complications of the operation.

Resection of localized pulmonary disease in patients with leukemia and aplastic anemia is feasible with acceptably low mortality and morbidity, and is often carried out for therapeutic as well as diagnostic reasons. Excessive perioperative blood loss caused by the aplastic state seldom occurs so long as platelets are administered. Lung tissue and bronchial stumps usually heal without difficulty.

Early mortality for lung biopsies in acute-onset diffuse pulmonary disease is high, and therapeutic benefit remains questionable in these patients, especially when broad empiric therapy has already been administered. In late-onset diffuse disease, patients may profit by lung biopsies, as directed change in therapy and positive effect of treatment are observed more often.

\section{REFERENCES}

1. Rosenow EC, Wilson WR, Cockerill FR. Pulmonary disease in the immunocompromised host. Mayo Clin Proc 1985;60: 473-87.

2. Speck B, Gratwohl A, Tichelli A, Nissen C. Knochenmarkstransplantation. Schweiz Med Wochenschr 1995;125:151532.

3. Tichelli A, Gratwohl A, Nissen C, Speck B. Late clonal complications in severe aplastic anemia. Leuk Lymphoma 1993; $12: 167-75$.

4. Doran HM, Sheppard MN, Collins PW, Jones L, Newland $\mathrm{AC}$, van der Walt JD. Pathology of the lung in leukemia and lymphoma: a study of 87 autopsies. Histopathology 1991;18: 211-9.

5. Sloane JP, Depledge MH, Powles RL, Morgenstern GR, Trickey BS, Dady PJ. Histopathology of the lung after bone marrow transplantation. J Clin Pathol 1983;36:546-54.

6. Shorter NA, Ross AJ, August $C$, et al. The usefulness of open-lung biopsy in the pediatric bone marrow transplant population. J Pediatr Surg 1988;23:533-7.

7. McCabe RE. Diagnosis of pulmonary infections in immunocompromised patients. Med Clin North Am 1988;72:1067-89.

8. Cheson BD, Samlowski WE, Tang TT, Spruance SL. Value of open-lung biopsy in 87 immunocompromised patients with pulmonary infiltrate. Cancer 1985;55:453-7.

9. Ostrow D, Buskard N, Hill RS, Vickars L, Churg A. Bronchiolitis obliterans complicating bone marrow transplantation. Chest 1985;87:828-30.

10. Perreault C, Cousineau S, D'Angelo G, et al. Lymphoid interstitial pneumonia after allogeneic bone marrow transplantation. Cancer 1985;55:1-9.

11. Crawford SW, Hackman RC, Clark JG. Biopsy diagnosis and clinical outcome of persistent focal pulmonary lesions after marrow transplantation. Transplantation 1989;48:266-71.

12. Young VK, Maghur HA, Luke DA, McGovern EM. Operation for cavitating invasive pulmonary aspergillosis in immunocompromised patients. Ann Thorac Surg 1992;55:563-4.

13. Lupinetti FM, Behrendt DM, Giller RH, Trigg ME, de Alarcon P. Pulmonary resection for fungal infection in children undergoing bone marrow transplantation. J Thorac Cardiovase Surg 1992;104:684-7.

14. Wong K, Waters CM, Walesby RK. Surgical management of invasive pulmonary aspergillosis in immunocompromised patients. Eur J Cardiothorac Surg 1992;6:138-43.

15. Moreau P, Zahar JR, Milpied N, et al. Localized invasive pulmonary aspergillosis in patients with neutropenia: effectiveness of surgical resection. Cancer 1993;72:3223-6. 